\title{
Peptide-Based Assemblies on Electrospun Polyamide-6/Chitosan Nanofibers for Detecting Visceral Leishmaniasis Antibodies
}

Michelle S. Liberato ${ }^{\dagger}$ Rodrigo S. N. Mancini, $^{\dagger}$ Irina M. Factori $^{\dagger}{ }^{\dagger}$ Fabio F. Ferreira, ${ }^{\dagger}$ Vivian L. de Oliveira, ${ }^{\ddagger}$ Juliana B. T. Carnielli, $₫$ Suchismita Guha, ${ }^{\S}$ Luis A. Peroni," Marcone A. L. Oliveira, ${ }^{\perp}$ Wendel A. Alves*,$\dagger$

${ }^{\dagger}$ Centro de Ciências Naturais e Humanas, Universidade Federal do ABC, 09210-580, Santo André, SP, Brazil

Núcleo de Doenças Infecciosas, Universidade Federal do Espírito Santo, 29047-100, Vitória, Espírito Santo, Brazil

$\S$ Department of Physics and Astronomy, University of Missouri, Columbia, MO 65211, USA

"Rheabiotech Company, 13084-791, Campinas, SP, Brazil

${ }^{\perp}$ Departamento de Química, Universidade Federal de Juiz de Fora, 36036-330, Juiz de Fora, MG, Brazil

Corresponding Authors

*E-mail:wendel.alves@ufabc.edu.br.Tel.:+551149960193. 


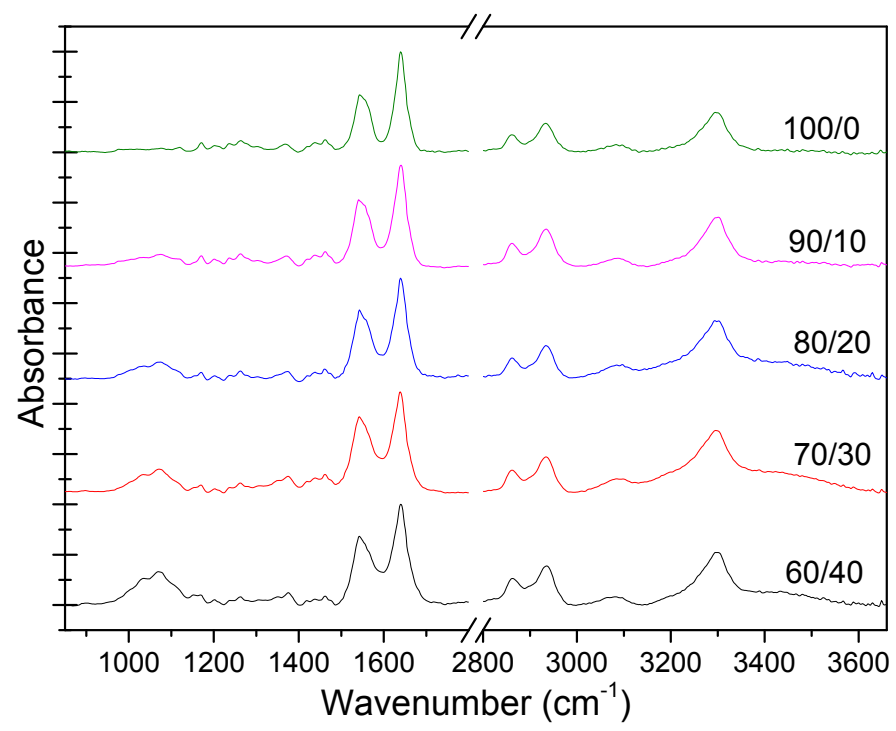

Figure S1. FTIR spectra of samples.

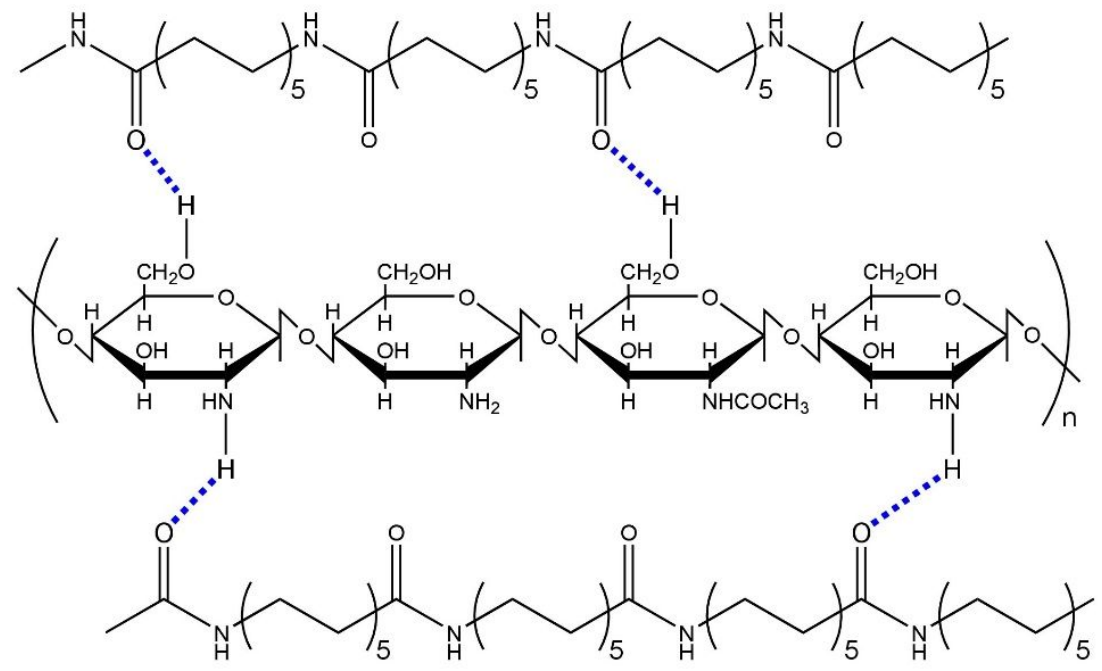

Figure S2. Proposed scheme of hydrogen bonding in PA6/chitosan blends. 

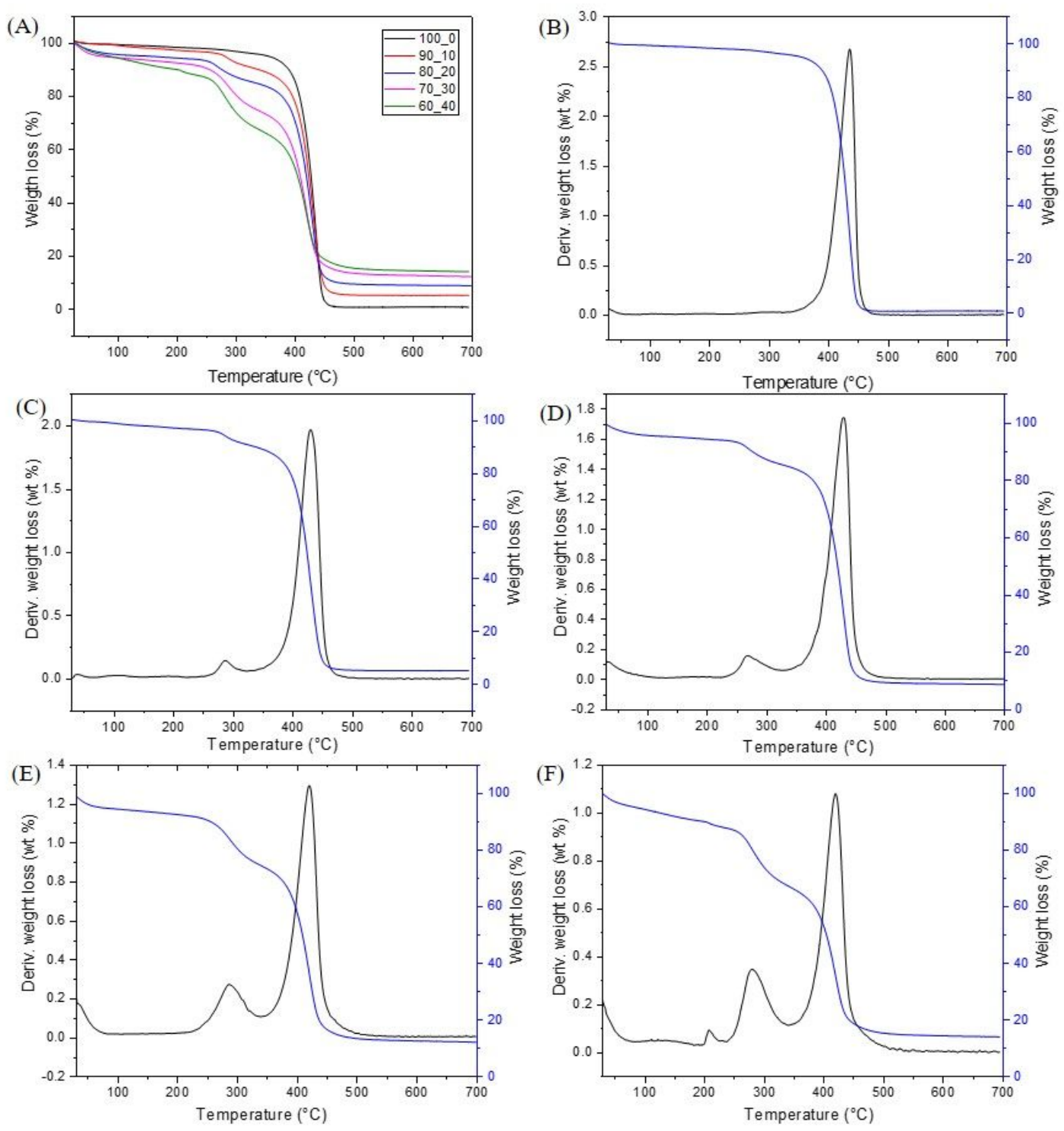

Figure S3. TGA and DTG curves of weight loss rate. TGA thermograms curves of weight loss rate by the initial sample mass at a heating rate of $10^{\circ} \mathrm{C} / \mathrm{min}$ in nitrogen condition (A) and (B-F) TG/ DTG blends ratio: 100/0, 90/10, 80/20, 70/30 and 60/40, respectively. 